\title{
Examples of integrable sub-Riemannian geodesic flows
}

\author{
Boris Kruglikov
}

\begin{abstract}
We exhibit examples of sub-Riemannian metrics with integrable geodesic flows and positive topological entropy.
\end{abstract}

\section{Introduction}

Consider a distribution on a manifold $M^{m}$, i.e. subbundle of the tangent bundle $\Pi \subset T M$. Non-holonomic Riemannian metric is a Riemannian metric $g \in S^{2} \Pi^{*}$ on this bundle. We call the pair $(\Pi, g)$ sub-Riemannian structure. A curve $\gamma:[0,1] \rightarrow M$ is called horizontal if $\dot{\gamma}$ is a section of $\Pi$. We denote the space of horizontal curves joining $x$ to $y$ by $\mathcal{H}(x, y)$. A theorem of Rashevsky-

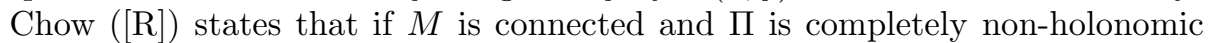
then $\mathcal{H}(x, y)$ is always non-empty. By completely non-holonomic we mean distribution $\Pi$, such that the module $\mathcal{D}_{\Pi}^{(N)}$ of order $\leq N$ self-commutators (of various kinds) of sections of $\Pi$ is equal to the module $\mathcal{D}(M)$ of all vector fields for some big $N$. From now on we consider only completely non-holonomic distributions.

For horizontal curves we calculate its length $l_{g}(\gamma)=\int_{0}^{1}\|\dot{\gamma}\|_{g} d t$ and this produces sub-Riemannian distance (metric) on $M$ by

$$
d_{g}(x, y)=\inf _{\gamma \in \mathcal{H}(x, y)} l_{g}(\gamma)
$$

A curve $\gamma \in \mathcal{H}$ is called geodesic if it realizes the minimum sub-distance for any two of its close points. The description of the most geodesics (normal ones) is given by the Euler-Lagrange variational principle. There is a Hamiltonian reformulation of this principle, due to Pontrjagin and co-authors PBGM, which allows to consider the geodesic flow as the usual Hamiltonian flow on $T^{*} M$. There appear occasionally geodesics of different kind - abnormals - which are not governed by the Pontrjagin principle for $\gamma$, but depend on the distribution $\Pi$ only. However if we consider contact distributions $\Pi$, i.e. distributions such that for any non-zero section $\alpha$ of the bundle $\operatorname{Ann}(\Pi) \subset T^{*} M$ we have $\alpha \wedge(d \alpha)^{n} \neq 0$ for $m=2 n+1$ (in particular $m=\operatorname{dim} M$ is odd), then all geodesics are normal.

As in the standard theory of geodesics we say the metric $g$ is integrable if the Hamiltonian flow of this metric is integrable on $T^{*} M$ in the Liouville sense, 
i.e. there are a.e. functionally independent integrals $I_{1}=H, I_{2}, \ldots, I_{n}$ which Poisson-commute $\left\{I_{k}, I_{l}\right\}=0$ (see BF] for a review of methods and problems).

For any diffeomorphism $f: M \rightarrow M$ the suspension $M \tilde{\times}_{f} S^{1}$ is the quotient of $M \times \mathbb{R}$ by the equivalence relation $(x, t) \sim(f(x), t+1)$. In particular for any matrix $A \in \mathrm{SL}_{n}(\mathbb{Z})$ we have a linear automorphism $A: T^{n} \rightarrow T^{n}$ of the torus and hence the manifold $M_{A}=T^{n} \tilde{\times}_{A} S^{1}$. In the paper [BT1] a series of examples of (Riemannian) geodesic flows on $M_{A}$ was constructed with the property that they are $C^{\infty}$-integrable, $C^{\omega}$ - (analytically) nonintegrable and have the topological entropy $h_{t o p}>0$.

We show that similar effect takes place in sub-Riemannian geometry too.

Theorem 1. For every $A \in \mathrm{SL}_{2}(\mathbb{Z})$ there are a contact distribution $\Pi$ on $M_{A}=T^{2} \tilde{\times}_{A} S^{1}$ and a non-holonomic Riemannian structure $g$ on it such that the sub-Riemannian geodesic flow is $C^{\infty}$-integrable.

In the case of real eigenvalues for $A \neq \pm E$ the flow is not integrable with geometrically simple set of integrals. Moreover the topological entropy is positive in semi-simple non-trivial case, $h_{\text {top }}=\max _{\lambda_{i} \in \operatorname{Sp}(A)}\left\{\ln \left|\lambda_{1}\right|, \ln \left|\lambda_{2}\right|\right\}$, and vanish otherwise, $h_{t o p}=0$.

The geometrically simple set of integrals includes analytic polynomial by momenta integrals and is defined in $\$$ (remark that we consider only integrability with Liouville tori, not cylinders, see $\S[(\mathrm{A})$ ).

Note that integrability of sub-Riemannian flow is a more complicated (and fascinating) fact since the description of the geodesics is completely nontrivial even locally (see $[\mathrm{VG}$ for a picture of sub-Riemannian wave front).

In particular locally over a point $x$ of the manifold Riemannian geodesic flow in $\tau_{M}^{-1}\left(U_{x}\right)$ is integrable, while in non-holonomic case it is no longer true. On the other hand integrability does not imply good behaviour in other respects: The well-known Martinet case (see ex-3) is $C^{\omega}$-integrable, but small geodesic balls are not sub-analytic ( $\mathrm{AS}]$ ) and there are abnormal geodesics.

Remark 1. One can easily generalize the above examples to the examples of integrable sub-Riemannian geodesic flows on $M_{A}$ for arbitrary $A \in \mathrm{SL}_{n}(\mathbb{Z})$ with $n>2$. There appear distributions of various ranks and kinds (they correspond to higher Vandermonde determinants instead of $2 \times 2$ matrices given below). Though completely non-holonomic, they have very degenerate curvature, i.e. the map $\Theta_{\Pi}: \Lambda^{2} \Pi \rightarrow \nu=T M / \Pi$ induced by commutators (in particular, the growth vector can be rather long). They never produce higher-dimensional contact examples.

Every sub-Riemannian metric $g$ on a manifold of $\operatorname{dim}=2 n+1$ produces canonically a transversal to the contact distribution $\Pi$ vector field - Reeb field $\nu_{\alpha}\left(\right.$ also denoted $R_{\alpha}$ ). To see it we choose $\alpha \in$ Ann $\Pi$ such that $\left\|\left.d \alpha\right|_{\Pi}\right\|=1$, where the norm is induced by the metric. In other words $\left.(d \alpha)^{n}\right|_{\Pi}=\operatorname{vol}_{g}$. This gives $\alpha$ uniquely for odd $n$ and up to \pm 1 for even if the distribution $\Pi$ is oriented. The Reeb field is defined now uniquely by $\alpha\left(\nu_{\alpha}\right)=1, i_{\nu_{\alpha}} d \alpha=0$. 
Theorem 2. In addition within the construction of theorem 1 if $\Pi$ is cooriented $\left(\mathrm{Sp} A\right.$ is contained in either $\mathbb{R}_{+}$or $\left.S^{1} \subset \mathbb{C}\right)$ the topological entropy of the Reeb flow is $h_{\text {top }}\left(\nu_{\alpha}\right)=0$.

We introduce Riemannian metric $\hat{g}$ on $M$ by the requirements $\left.\hat{g}\right|_{\Pi}=g$, $\nu_{\alpha} \perp_{\hat{g}} \Pi,\left\|\nu_{\alpha}\right\|_{\hat{g}}=1$.

Theorem 3. The geodesic flow of Riemannian metric $\hat{g}$ on $M_{A}$ is $C^{\infty}$ integrable, $C^{\omega}$-nonintegrable for real eigenvalues of $A \neq \pm E$ and has positive topological entropy for semi-simple nontrivial case.

In fact the metric $\hat{g}$ coincides with the metric on $M_{A}$ introduced by Bolsinov and Taimanov. The first example of $C^{\infty}$-integrable geodesic flow violating obstructions for $C^{\omega}$-integrability was found by Butler [B] and then it was included into a much bigger family of examples-suspensions in [BT1]. Their examples obviously generalize to Lorentian and other semi-geometries. We show the case of sub-Riemannian geometry (actually of any dim) can be considered as well.

Remark 2. Note that examples from [BT1] are easily generalized to a larger group $\mathrm{GL}_{n}(\mathbb{Z})=\mathrm{SL}_{n}(\mathbb{Z}) \times \mathbb{Z}_{2}$. However the sub-Riemannian case is different: the manifold $M_{A}$ with $\operatorname{det} A=-1$ does not admit any contact structure. Actually a contact structure on a 3-dimensional manifold gives a canonical orientation.

We discuss some relations between $g, \hat{g}$ and $\nu_{\alpha}$ at the end of the paper. In $\S$ 团 we exibit Poisson action of $\mathbb{R}^{3}$ such that entropy of every non-zero vector is non-zero.

Let us note that theorem 1 provides the first example of $C^{\omega}$-polynomial in momenta nonintegrable sub-Riemannian geodesic flow. In paper MSS it was presented only algebraically non-integrable sub-Riemannian flow on a Lie group of $\operatorname{dim} M=6$. In dimension 3 all left-invariant sub-geodesic flows on Lie groups are Liouville integrable (as we show in $\delta$ 㑑).

Acknowledgements. I would like to thank J. P. Gauthier, A. V. Bolsinov, V.S. Matveev and O. Kozlovski for helpful discussions. I am indebted to A. B. Katok for the reference in $\S 3$. My interest to sub-Riemannian geometry has grown up during a Singularity Theory workshop at Newton Institute (Cambridge), 2000. I would like to thank the Institute and especially V. V. Goryunov and V. M. Zakalyukin for the organization of this activity and hospitality.

\section{Sub-Riemannian geometry and Hamiltonian systems}

\section{(A) Integrable Hamiltonian flows.}

Let $\left(W^{2 n}, \Omega\right)$ be a symplectic manifold. Hamiltonian vector field is the field $\Omega$-dual to an exact 1-form $d H: \Omega\left(X_{H}, \cdot\right)=d H$. We denote the field $X_{H}$ also by 
sgrad $H$, since the above is similar to the definition of the usual gradient (and in order not to confuse with $M_{A}$ above).

Poisson brackets can be defined as $\{F, G\}=\Omega\left(X_{F}, X_{G}\right)$. The Hamiltonian system is called complete (or Liouville) integrable if there are additionally to $I_{1}=H$ involutive integrals $I_{2}, \ldots, I_{n},\left\{I_{j}, I_{k}\right\}=0$, which are functionally independent a.e. By Liouville theorem ([A]) a full measure set $W^{\prime} \subset W$ is then foliated by cylinders (tori in compact case), and each cylinder has a neighborhood with coordinates $\varphi \in T^{n-r} \times \mathbb{R}^{r}$ and new $I \in \mathbb{R}^{n}$ s.t. $\Omega=d I \wedge d \varphi$, $\left\{I_{j}=c_{j}\right\}_{1 \leq j \leq n} \simeq T^{n-r} \times \mathbb{R}^{r}$ and the flow is $\dot{\varphi}=\omega(I)$. The number $r$ (noncompactness rank) vanishes in many important examples. We will always suppose $r=0$ (even for noncompact $W$ ) and call this case Liouville integrability.

Let $(M, g)$ be a Riemannian manifold. Then the geodesic flow $\varphi_{t}: T M \rightarrow$ $T M$ can be considered as Hamiltonian on $T^{*} M \stackrel{\sharp^{g}}{\simeq} T M$ with the standard symplectic structure $\Omega$ if we choose the Hamiltonian $H=\frac{1}{2}\|p\|^{2}, p \in T^{*} M$. We say $g$ is integrable if the flow $\varphi_{t}$ is Liouville-integrable.

\section{(B) Pontrjagin maximum principle.}

Consider now non-holonomic case. We start with an arbitrary completely non-holonomic distribution $\Pi \subset T M$.

Let $\left(T^{*} M, \Omega\right)$ be the cotangent bundle equipped with the standard symplectic structure. The non-holonomic metric defines isomorphism $\sharp^{g}: \Pi^{*} \rightarrow \Pi$. Consider the inclusion $i: \Pi \hookrightarrow T M$. Then we have vector bundles morphism $\Psi_{g}$ defined as the following composition:

$$
T^{*} M \stackrel{i^{*}}{\rightarrow} \Pi^{*} \stackrel{\sharp^{g}}{\rightarrow} \Pi \stackrel{i}{\rightarrow} T M .
$$

Contrary to Riemannian situation this is not isomorphism. We have: $\operatorname{Ker}\left(\Psi_{g}\right)=$ Ann $\Pi$ and $\operatorname{CoKer}\left(\Psi_{g}\right)=\nu=T M / \Pi$.

Define the Hamiltonian function $H$ on $T^{*} M$ as the composition

$$
T^{*} M \stackrel{i^{*}}{\rightarrow} \Pi^{*} \stackrel{\sharp g}{\rightarrow} \Pi \stackrel{\frac{1}{2}\|\cdot\|_{g}^{2}}{\rightarrow} \mathbb{R}
$$

This function can be locally described as follows. Let $\xi_{1}, \ldots, \xi_{k}$ be some orthonormal basis of vector fields tangent to $\Pi$. Every vector field is a fiber-linear function on $T^{*} M$. So we have $H=\frac{1}{2} \sum_{1}^{k} \xi_{i}^{2}$.

The Pontrjagin maximum principle ([PBGM $)$ states that trajectories of this vector fields in region $\{H>0\}$ projected to $M$ are optimal for the corresponding variational problem. They are called (normal) geodesics.

Example 1. Consider $M=T^{3}=\mathbb{R}^{3} / 2 \pi \mathbb{Z}^{3}$ with cyclic coordinates $\left(\varphi_{1}, \varphi_{2}, \varphi_{3}\right)$. Let $\alpha=\sin \varphi_{1} d \varphi_{2}+\cos \varphi_{1} d \varphi_{3}$ be a contact form and $\Pi=$ Ker $\alpha$ the corresponding distribution. Let the metric be induced from the standard metric $d s^{2}=\sum d \varphi_{i}^{2}$ on the torus. Then $H=\frac{1}{2}\left[p_{1}^{2}+\left(\cos \varphi_{1} p_{2}-\sin \varphi_{1} p_{3}\right)^{2}\right]$ and the 
Hamilton equations $\dot{\varphi}=\partial H / \partial p, \dot{p}=-\partial H / \partial \varphi$ have the form:

$$
\begin{aligned}
& \dot{\varphi}_{1}=p_{1}, \quad \dot{\varphi}_{2}=\cos ^{2}\left(\varphi_{1}\right) p_{2}-\frac{\sin \left(2 \varphi_{1}\right)}{2} p_{3}, \quad \dot{\varphi}_{3}=-\frac{\sin \left(2 \varphi_{1}\right)}{2} p_{2}+\sin ^{2}\left(\varphi_{1}\right) p_{3}, \\
& \dot{p}_{1}=\frac{\sin \left(2 \varphi_{1}\right)}{2}\left(p_{2}^{2}-p_{3}^{2}\right)+\cos \left(2 \varphi_{1}\right) p_{2} p_{3}, \quad \dot{p}_{2}=0, \quad \dot{p}_{3}=0 .
\end{aligned}
$$

They can be easily integrated. The functions $I_{2}=p_{2}, I_{3}=p_{3}$ are obviously integrals. This can be also checked via Poisson brackets: the functions $I_{1}=H$, $I_{2}, I_{3}$ are involutive: $\left\{I_{j}, I_{k}\right\}=0$. The only singularities are $\odot$ and $\bigcirc$ (socalled atoms $A$ and $C_{2}$ ).

Example 2. The geodesics on the Heisenberg group are given by the $2 H=$ $\left(p_{1}+x_{3} p_{2}\right)^{2}+p_{3}^{2}$. Solving the Hamiltonian equation we see that geodesics are spirals in the direction of $x_{2}$-axis, projecting to arbitrary (including radius $\infty$ ) circles on the plane $\mathbb{R}^{2}\left(x_{1}, x_{3}\right)$.

\section{(C) Note on abnormals.}

In some situations there are optimal lines, which depend on the distribution $\Pi$ only. They are called abnormal geodesics and in generic case are described as follows.

Consider the submanifold $S=\operatorname{Ann} \Pi \subset T^{*} M$. It is given by equation $H=0$. So contrary to the Riemannian case $k=\operatorname{codim} S=\operatorname{dim} \Pi<m=\operatorname{dim} M$. Let $0_{M} \subset T^{*} M$ be the zero section and $S_{0}=S \backslash 0_{M}$ be the complement. This $S_{0}$ is a punctured cone over $0_{M}$.

Let $k \in 2 \mathbb{Z}$. Then generically there is a hypersurface $\Sigma^{2 n-k-1} \subset S_{0}$ (maybe empty), where $\left.\Omega^{n-k / 2}\right|_{S_{0}}=0$. The integral lines of $\operatorname{Ker}\left(\left.\Omega\right|_{\Sigma}\right)$ projects to the abnormals.

Let $k \in\{2 \mathbb{Z}+1\}$. Then generically kernels of $\left.\Omega\right|_{S_{0}}$ are one-dimensional, so they integrates to lines projecting to the abnormals.

This geometric description comes from the variational approach of Hsu.

Example 3. Consider the Martinet case: $\Pi=\operatorname{Ker} \alpha$ for $\alpha=d y-z^{2} d x$ on $\mathbb{R}^{3}(x, y, z)$. Let the metric be lifted from the Euclidean plane $\mathbb{R}^{2}(x, z)$ (recall the abnormals do not depend on a choice of the metric). So $2 H=\left(p_{x}+z^{2} p_{y}\right)^{2}+p_{z}^{2}$. There is no flow on $S=\{H=0\}$ (as usual because $H$ is quadratic). However there are geodesics.

Actually, $S=\left\{p_{x}=-z^{2} p_{y}, p_{z}=0\right\}$. So the coordinates are $\left(x, y, z, p_{y}\right)$. Since $\Omega=d x \wedge d p_{x}+d y \wedge d p_{y}+d z \wedge d p_{z}$ we get $\left.\Omega^{2}\right|_{S}=2 z p_{y} d x \wedge d y \wedge d z \wedge d p_{y}$. Since on $S_{0} p_{y} \neq 0$ we conclude that $\Sigma^{3}=\{z=0\} \subset S_{0}$ with coordinates $\left(x, y, p_{y}\right)$. Now $\left.\Omega\right|_{\Sigma}=d y \wedge d p_{y}$, whence $\operatorname{Ker} \Omega=\left\langle\partial_{x}\right\rangle$ and the abnormal geodesics are given by $\{y=$ const, $z=0\}$. 
Example 4. Consider the Engel distribution: Ann $\Pi=\langle d y-z d x, d z-w d x\rangle$, i.e. $\Pi=\left\langle\partial_{x}+z \partial_{y}+w \partial_{z}, \partial_{w}\right\rangle$ on $\mathbb{R}^{4}(x, y, z, w)$. The manifold $S$ is given by $\left\{p_{x}=-z p_{y}-w p_{z}, p_{w}=0\right\}$ and has coordinates $\left(x, y, z, w, p_{y}, p_{z}\right)$. So $\left.\Omega^{3}\right|_{S_{0}}=0$ is equivalent to $p_{z}=0$. This gives the submanifold $\Sigma^{5}$ and $\left.\Omega\right|_{\Sigma}=-p_{y} d x \wedge d z-$ $z d x \wedge d p_{y}+d y \wedge d p_{y}$, whence $\operatorname{Ker}\left(\left.\Omega\right|_{\Sigma}\right)=\left\langle\partial_{w}\right\rangle$. This is so-called characteristic direction for the Engel distribution and the abnormals are just the corresponding integral curves.

It follows from the above description that non-holonomic metrics on contact distributions have no abnormals.

\section{(D) Local integrability.}

Though in many respects sub-Riemannian structures are similar to Riemannian ones (e.g. $\mathrm{ACG}$ ), as the previous subsection shows there are differences. Another discriminating aspect is Liouville integrability.

Let us consider Euclidean metric locally in a neighborhood $B_{\varepsilon}(x)$. Symplectic reduction of the corresponding Hamiltonian flow on $\{H=1 / 2\}$ is $T_{<\varepsilon}^{*} S^{n-1}$. By convexity theorem for arbitrary Riemannian metric and small $\varepsilon$ the symplectic reduction preserves differentiable type of $T^{*} S^{n-1}$. Moreover by Moser isotopy method applied to (open) neighborhood of the zero section we get that germs of reductions are symplectomorphic in Riemannian and linearized (Euclidean) cases. So one can find involute set of functions and integrate (by Liouville) the Riemannian geodesic flow on a neighborhood $U_{x} \subset B_{\varepsilon}(x)$.

In contrast even the differentiable type of symplectic reduction for subRiemannian structure in the simplest Heisenberg case can be changed under arbitrary small perturbation. That is because the geodesics are curved and being perturbed may leave/return the domain.

\section{Entropy of a dynamical system}

\section{(A) Definition of entropies.}

Consider at first discrete time dynamical systems. Let $M$ be a compact topological space with probability measure $\mu$ and $f: M \rightarrow M$ be a homeomorphism. Consider some partition $\xi$ (up to zero measure) $M=\sqcup \xi_{i}$ of our space by positive-measure sets with finite or countable number of indices. We form the new partition $\xi_{-n}^{f}=\bigvee_{i=0}^{n} f^{-i} \xi$, where $\xi \vee \eta$ is a partition formed by the sets $\xi_{\alpha} \cap \eta_{\beta}$.

We define ( $\mathrm{KH}$ ) entropy of the partition by $H(\xi)=-\sum \mu\left(\xi_{j}\right) \ln \mu\left(\xi_{j}\right)$ and entropy of a preserving measure map $f$ w.r.t. $\xi$ by $h_{\mu}(f ; \xi)=\lim _{n \rightarrow \infty} \frac{H\left(\xi_{-n}^{f}\right)}{n}$. Then we define measure entropy of $f$ by

$$
h_{\mu}(f)=\sup _{\left\{\xi \mid h_{\mu}(f ; \xi)<\infty\right\}} h_{\mu}(f ; \xi) .
$$


To define topological entropy we should change partition $\xi$ by an open cover $\mathfrak{U}$ of $M=\cup U_{j}$, function $H(\xi)$ by the function $N(\mathfrak{U})$ that is cardinality of minimal subcovering and then take sup over all open coverings. This quantity is subject to the following variational principle:

$$
h_{\mathrm{top}}(f)=\sup _{\mu} h_{\mu}(f),
$$

where the supremum is taken over all $f$-invariant Borel probability measures on $M$.

Another useful definition of the topological entropy goes for metric spaces $(M, d)$. We define $d_{n}^{f}=\max _{0 \leq i \leq n-1}\left(f^{i}\right)^{*} d$ and denote by $S(f, \varepsilon, n)$ the minimal number of $\varepsilon$-balls in $d_{n}^{f}$-metric to cover $M$. Then

$$
h_{\mathrm{top}}(f)=\lim _{\varepsilon \rightarrow 0} \varlimsup_{n \rightarrow \infty} \frac{\ln S(f, \varepsilon, n)}{n}=\lim _{\varepsilon \rightarrow 0} \varliminf_{n \rightarrow \infty} \frac{\ln S(f, \varepsilon, n)}{n} .
$$

This limit depends not on $d$ but on the $d$-topology only $([\mathrm{KH}])$.

Example 5. Let $A \in \mathrm{GL}_{n}(\mathbb{Z})$. Then we can define map $A: T^{n} \rightarrow T^{n}$, where $T^{n}=\mathbb{R}^{n} / \mathbb{Z}^{n}$. Let the measure be $d \mu=d x_{1} \wedge \ldots \wedge d x_{n}$ in the standard coordinates. Then

$$
h_{\text {top }}(A)=h_{\mu}(A)=\sum_{\lambda_{A} \in \mathrm{Sp}^{+}(A)} \ln \left|\lambda_{A}\right|,
$$

where $\mathrm{Sp}^{+}(A)$ is the part of the spectrum outside the unit disk $D_{1} \subset \mathbb{C}$.

If $v \in \mathcal{D}(M)$ is a vector field on a manifold $M$ we define $h_{\mathrm{top}}(v)=h_{\mathrm{top}}\left(\varphi_{v}^{1}\right)$, where $\varphi_{v}^{t}: M \rightarrow M$ is the flow of $v$. We define the measure entropy similarly and note that for continuous time dynamical systems the entropies are also connected by the variational principle.

\section{(B) Compactness restriction.}

If $M$ is noncompact, the previous definitions basically do not work. Still it is possible to define the entropy.

The easiest case is as follows. Suppose there exists a compact exhaustion $M=\cup_{j=1}^{\infty} K_{j}$ with $K_{j} \subset K_{j+1}$ and each $K_{j}$ being compact and $f$-invariant. Then we define topological entropy by

$$
h_{\text {top }}(f)=\lim _{j \rightarrow \infty} h_{\text {top }}\left(\left.f\right|_{K_{j}}\right) \in \mathbb{R}_{\geq 0} \cup\{+\infty\} .
$$

Since the sequence $h_{\text {top }}\left(\left.f\right|_{K_{j}}\right)$ is non-decreasing the limit exists and since it exists for every exhaustion $\left\{K_{j}\right\}_{1}^{\infty}$ it does not depend on its choice. We call such case non-compactness of the first type. 
As an example we consider Liouville integrable Hamiltonian system sgrad $H$ with commuting integrals $I_{1}=H, I_{2}, \ldots, I_{n}$ on $M^{2 n}$, for which generic intersection $\left\{I_{j}=\right.$ const $\left._{j}\right\}$ is a union of tori. Then we obviously have invariant compact exhaustion and define $h_{\text {top }}(\operatorname{sgrad} H)$.

In the general situation we consider arbitrary compact exhaustion $M=\cup K_{j}$ (not necessarily $f$-invariant). Let us study the restriction of the metric $d_{n}^{f}$ to $K_{j}$. Since $K_{j}$ is compact there is a finite covering by $\varepsilon$-balls. Calculating the asymptotic of the minimal cardinality of such a cover we get similarly to (11) the quantity $h_{\mathrm{top}}\left(f ; K_{j}\right)$. This sequence is non-decreasing by $j$ and we set

$$
h_{\mathrm{top}}(f)=\lim _{j \rightarrow \infty} h_{\mathrm{top}}\left(f ; K_{j}\right) .
$$

Again the limit does not depend on a choice of the compact exhaustion. But it can be infinite even for sufficiently smooth $f$. Let's call the situation noncompactness of the second type.

Example 6. Consider a linear automorphism $A: \mathbb{R}^{n} \rightarrow \mathbb{R}^{n}$. Then the entropy $h_{\text {top }}(A)$ is given by the same formula (6) as in example 5 .

Note that this example shows $h_{\text {top }}\left(f^{-1}\right) \neq h_{\text {top }}(f)$ generally in non-compact case. However all properties of the entropy holds for non-compactness of the first type.

The measure analogs of the above definitions are straightforward.

\section{(C) Lyapunov exponents.}

Let $\|\cdot\|$ be any norm on $T M$ and $f: M \rightarrow M$ be of class $C^{1}$. Then we can consider the map $v \mapsto \varlimsup_{k \rightarrow \infty} \frac{\ln \left\|f_{*}^{k} v\right\|}{k}$. This map takes values $\chi_{1}(x) \leq \cdots \leq$ $\chi_{n}(x)$ at (almost) any point $x \in M^{n}$, which are called Lyapunov exponents (multiple values repeat). They are defined similarly for the flows and do not depend on a choice of the norm defining the topology.

Ruelle inequality says that if $\mu$ is $f$-invariant Borel probability measure, then

$$
h_{\mu}(f) \leq \int_{M}\left[\sum_{\chi_{j}(x)>0} \chi_{j}(x)\right] d \mu .
$$

In particular if the norm can be chosen so that $\left\|\left(\varphi_{t}\right)_{*} v\right\|$ is constant (Lebesgue) a.e., then the entropy vanishes w.r.t. any Liouville (i.e. absolutely continuous) invariant probability measure. Therefore we get

Example 7. If a Hamiltonian system with Hamiltonian $H$ is completely integrable, then for every Liouville invariant measure $h_{\mu}(\operatorname{sgrad} H)=0$.

Moreover Pesin theorem states that the inequality above becomes equality

$$
h_{\mu}(f)=\int_{M} \sum_{\chi_{j}>0} \chi_{j}(x) d \mu
$$


for $C^{1+\varepsilon}$-diffeomorphisms/flows and any Liouville measure.

Since for integrable Hamiltonian system every measure $\rho\left(I_{1}, \ldots, I_{n}\right)\left|\Omega^{n}\right|$ is invariant, where $\Omega$ is the symplectic form and $I_{j}$ are integrals, there are finite Liouville measures in this case (even if $M^{2 n}$ is non-compact).

\section{(D) Geodesic flows.}

Let $(M, g)$ be a compact Riemannian manifold. Let $\varphi_{t}: T M \rightarrow T M$ be the geodesic flow. Note that restriction of this flow to invariant compact submanifold $T_{c} M=\left\{v \in T M,\|v\|_{g}=c\right\}$ is conjugated to $\left.\varphi_{t}\right|_{T_{1} M}$. Hence we define entropy $h(g)$ of the metric $g$ to be the entropy of the last flow.

Note that since the geodesic flow is Hamiltonian the (Liouville) measure entropy vanishes $h_{\mu}(g)=0$ for an integrable metric $g$ due to example 7 . The behavior of topological entropy as we will see in $\$$ 的 can be more complicated.

Example 8. Geodesic flow on the group $\mathrm{SO}(3)$ with left-invariant Riemannian metric has $h_{\text {top }}(g)=0$.

Remark that canonical identification $T^{*} M \stackrel{\sharp^{g}}{\simeq} T M$ allows to consider the lifted flow $\varphi_{t}: T^{*} M \rightarrow T^{*} M$. Its restriction to the hypersurface $T^{1} M=\{H=$ $1 / 2\}$ determines the entropy, where $H$ is the Hamiltonian of the geodesic flow (for non-compact $M$ we should use the above modification of $h_{\text {top }}$ ).

Consider now sub-Riemannian structure $(M, \Pi, g)$. We again can consider geodesic flow and restrict it to the isoenergetic surface $Q=\{H=1 / 2\} \subset T^{*} M$, $\varphi_{t}: Q \rightarrow Q$. This $Q$ however is always non-compact, so that we should use the non-compact version of the entropy: $h_{\mathrm{top}}(\Pi, g):=h_{\mathrm{top}}\left(\left.\varphi_{t}\right|_{Q}\right)$.

Example 9. For the group $\mathrm{SO}(3)$ the topological entropy of sub-Riemannian geodesic flow (see $\$$ 团) is zero. This follows from the study of bifurcational diagram (also directly from the description of the geodesics in $[\mathrm{VG}]$ ).

Note that if the Hamiltonian flow sgrad $H$ is Liouville-integrable we have non-compactness of the first type, Lyapunov exponents vanish a.e. due to Liouville theorem and the (Liouville) measure entropy is zero.

\section{Commuting dynamical systems}

Let $f, g: M \rightarrow M$ be two commuting dynamical systems. They define an action of $\mathbb{Z}^{2}$ on $M$. If we have more commuting systems or commuting flows, then higher dimensional groups $\mathbb{Z}^{k}$ or $\mathbb{R}^{k}$ act. There is a definition of entropy of such an action $(\mathbb{S} \mid)$. However if it is non-trivial, then entropies of all dynamical systems given by 1-dimensional subgroups are infinite. We consider instead the case when the higher entropy is zero and the entropies of the generators of the group are finite. 
Theorem 4 ([ $[\mathrm{Hu}])$. Consider a $\mathbb{Z}^{2}$-action with generators $f, g$ of class $C^{1+\varepsilon}$. Let $h$ be either $h_{\mu}$, where $\mu$ is any $\mathbb{Z}^{2}$-invariant Borel probability measure on $M$, or $h_{\text {top }}$ and then we additionally suppose $f g \in C^{\infty}$. Then:

$$
h(f g) \leq h(f)+h(g) .
$$

Proof. Let us indicate the proof for a Liouville measure. Let $\chi_{i}^{f}(x)$ and $\chi_{j}^{g}(x)$ be Lyapunov exponents for $f$ and $g$ respectively. Take $\chi_{i}^{f}(x)<\lambda<$ $\chi_{i+1}^{f}(x)$. Then the map $F_{\lambda}:=\lambda^{-1} f_{*}: T M \rightarrow T M$ commutes with $g_{*}$ and so $\left(F_{\lambda}\right)^{r} \circ g_{*}=g_{*} \circ\left(F_{\lambda}\right)^{r}$. Tending $r \rightarrow \pm \infty$ we get $g$-invariance of the repellingexpanding directions decomposition $T_{x} M=H_{i}^{-} \oplus H_{i}^{+}$.

Applying this to all $\lambda$ different from Lyapunov exponents and interchanging $f$ and $g$ we deduce for $x$ from a full measure subset in $M$ the common decomposition $T_{x} M=\oplus H_{s}(x)$ which is: measurable, invariant and such that on every subspace $H_{s}$ the Lyapunov exponents of both $f$ and $g$ are constant. Therefore the Lyapunov exponents of $f \circ g$ are sums of the corresponding Lyapunov exponents for $f$ and $g$.

The claim now follows from the Pesin formula $1(\mathrm{C})$. The equality in (3) is achieved if and only if we sum positive exponents for $f$ and $g$ in the above decomposition $\oplus H_{i}$ almost everywhere w.r.t. $\mu$.

The case of general Borel measure as well as the inequality for $h_{\text {top }}$ is done in $\mathrm{Hu}$ using Lyapunov charts (theorems B,C).

Remark 3. Without commutativity assumption the statement is false. Actually the Anosov automorphism $A: T^{2} \rightarrow T^{2}$ with $A=\left(\begin{array}{ll}2 & 1 \\ 1 & 1\end{array}\right)$ can be decomposed $A=B B^{t}$ with $B=\left(\begin{array}{ll}1 & 1 \\ 0 & 1\end{array}\right)$. But $h_{\text {top }}(A)=\ln \frac{3+\sqrt{5}}{2}$, while $h_{\text {top }}(B)=$ $h_{\text {top }}\left(B^{t}\right)=0$.

Also the requirement that the dynamical systems are smooth is crucial: There are continuous $\mathbb{Z}^{2}$-actions ([Pa]) with vanishing directional entropies for all irrational directions and non-zero for all rational.

Corollary 1. Let $\mathbb{Z}^{2}$-dynamical system given by smooth commuting $f, g$ be either defined on a compact manifold or have non-compactness of the first type. Let $h$ denote $h_{\mu}$ or $h_{\text {top }}$. If $h(g)=0$, then $h(f g)=h(f)$.

Proof. Actually $h(f g) \leq h(f)$ and using $h\left(g^{-1}\right)=h(g)=0$ we get the reverse inequality.

Similar to (3) formula holds for commuting flows $\varphi_{\xi}^{t}, \varphi_{\eta}^{s}$ generated by vector fields $\xi, \eta$ :

$$
h\left(\varphi_{\xi}^{t} \varphi_{\eta}^{s}\right) \leq h\left(\varphi_{\xi}^{t}\right)+h\left(\varphi_{\eta}^{s}\right),
$$

where $h=h_{\text {top }}$ or $h_{\mu}$ as in the theorem. 
Therefore if $\mathbb{R}^{k}$ acts on $M$ with generators $\varphi_{1}^{t_{1}}, \ldots, \varphi_{k}^{t_{k}}$ we define

$$
\rho(v)=h\left(\varphi_{1}^{t_{1}} \cdots \varphi_{k}^{t_{k}}\right) \text { for } v=\left(t_{1}, \ldots, t_{k}\right) \in \mathbb{R}^{k} .
$$

The inequality (4) can be seen now as triangle inequality and hence $\rho$ is a pseudonorm on $\mathbb{R}^{k}$ (provided non-compactness is no greater than the first type). This $\rho$ however should not be continuous and can be degenerate (not norm).

\section{Integrability of sub-Riemannian metrics on 3- dimensional Lie groups}

Consider 3-dimensional Lie groups $G$. Every left-invariant structure is presented on the Lie algebra $\mathcal{G}$. So we write the classification of left-invariant non-holonomic metrics on contact structures along Bianchi classification of 3dimensional Lie algebras ( $\mathrm{VG})$. We identify proportional metrics (the geodesic flows are reparameterized). We present distribution $\Pi \subset \mathcal{G}$ and the metric by an orthonormal basis $\xi_{1}, \xi_{2}$ :

1. Heisenberg algebra $\mathfrak{h}(3):\left[e_{1}, e_{2}\right]=e_{3},\left[e_{k}, e_{3}\right]=0$ and $\xi_{k}=e_{k}, k=1,2$.

2. Solvable algebras: $\left[e_{1}, e_{2}\right]=0$,

(a) $\left[e_{1}, e_{3}\right]=\lambda_{1} e_{1},\left[e_{2}, e_{3}\right]=\lambda_{2} e_{2}\left(\lambda_{1} \neq \lambda_{2}\right)$ and $\xi_{1}=e_{1}+e_{2}, \xi_{2}=e_{3}$.

(b) $\left.\left[\left(e_{1}+i e_{2}\right), e_{3}\right)\right]=e^{-i \varphi}\left(e_{1}+i e_{2}\right)(0<\varphi<\pi)$ and $\xi_{1}=e_{1}, \xi_{2}=e_{3}$.

(c) $\left[e_{1}, e_{3}\right]=e_{1}+e_{2},\left[e_{2}, e_{3}\right]=e_{2}$ and $\xi_{1}=e_{1}, \xi_{2}=e_{3}$.

3. Orthogonal algebra so(3): $\left[e_{1}, e_{2}\right]=e_{3},\left[e_{2}, e_{3}\right]=e_{1},\left[e_{3}, e_{1}\right]=e_{2}$ and $\xi_{1}=e_{1}, \xi_{2}=\sigma e_{2}$.

4. Special linear algebra $\operatorname{sl}_{2}(\mathbb{R}):\left[e_{1}, e_{2}\right]=e_{3},\left[e_{1}, e_{3}\right]=2 e_{1},\left[e_{2}, e_{3}\right]=-2 e_{2}$ and two possibilities

(a) $\xi_{1}=e_{1}, \xi_{2}=\sigma e_{2}$;

(b) $\xi_{1}=e_{1}+e_{2}, \xi_{2}=\sigma e_{3}$.

In three last cases the parameter $\sigma \in \mathbb{R}_{+}$.

One can integrate the equations of geodesics (see $\mathbb{V G}$ for a description in terms of semi-direct product). We consider Liouville integrability.

Theorem 5. Non-holonomic geodesic flows of the above metrics on 3-dimensional Lie groups are $C^{\omega}$-integrable.

Proof. First note that any vector $v \in \mathcal{G}$ generates left- and right-invariant vector fields $L_{v}$ and $R_{v}$ on $G$, which can be considered as functions on $T^{*} G$. We also denote by $L_{v}, R_{v} \in C^{\infty}\left(T^{*} G\right)$ the invariant extensions of any function $v \in C^{\infty}\left(\mathcal{G}^{*}\right)$, not necessarily linear. Any two $L_{v}$ and $R_{w}$ Poisson commute and 
the other Poisson brackets are $\left\{L_{v}, L_{w}\right\}=L_{[v, w]},\left\{R_{v}, R_{w}\right\}=R_{[v, w]}$ (AKN, [F]), where the bracket [,] on $C^{\infty}\left(\mathcal{G}^{*}\right)$ is induced by the usual commutator (this bracket is called Lie-Poisson or Berezin-Kirillov-Konstant-Souriau).

So finding one left-invariant function $I_{2}=L_{v}$ commuting with $2 H=\xi_{1}^{2}+\xi_{2}^{2}$ will suffice, just take any $I_{3}=R_{w}$. We take the Casimir function $F$ for $v$, i.e. such a function that $[\cdot, F] \equiv 0$. This function always exists locally since $\mathcal{G}$ has odd dimension, but globally it can have singularities.

In the case of so(3) we have smooth Casimir function $F=e_{1}^{2}+e_{2}^{2}+e_{3}^{2}$. For $\operatorname{sl}_{2}(\mathbb{R}) F=4 e_{1} e_{2}-e_{3}^{2}$. For the Heisenberg group $F=e_{3}$. For the solvable group we consider $\left[\cdot, e_{3}\right]$ as a linear vector field on the plane $\mathbb{R}^{2}\left(e_{1}, e_{2}\right)$. We take $F$ to be an integral of this vector field. For example for the case (3a) we take $F=\left(e_{1}\right)^{\lambda_{2}}\left(e_{2}\right)^{-\lambda_{1}}$.

Note that these functions $F$ have singularities along some axes in the solvable case. To overcome this difficulty we use the lemma.

Lemma 6. If 3-dim Lie group $G$ is not semisimple, then for every left-invariant $H$ there exist two commuting right-invariant integrals $I_{2}, I_{3}$.

Actually due to Bianchi classification we can always find a two-dimensional commutative subalgebra $\langle v, w\rangle \subset \mathcal{G}$ in non-semisimple case $\left(\left\langle e_{1}, e_{2}\right\rangle\right.$ for solvable algebras). Now it's easy to check that in every case of non-holonomic metric we obtain 3 functionally independent a.e. integrals.

Note on the non-holonomic flows on $\mathrm{SL}_{2}(\mathbb{R})$. The two contact structures on $\mathrm{SL}_{2}(\mathbb{Z})$ have nice geometric interpretations $([\mathrm{Kr}])$. It is well known that $\mathrm{SL}_{2}(\mathbb{Z}) /\{ \pm 1\} \simeq S T^{*} L^{2}$ for the spherical bundle of the Lobachevskii plane $L^{2}$ and since every surface $M^{2}$ of genus $g>1$ can be obtained as quotient of $L^{2}$ by a discrete subgroup we end up with two contact structures on $S T^{*} M^{2}$ of which one is the standard and the other is the connection form associated with a metric of constant negative curvature.

Note that the metric descends to $S T^{*} M^{2}$ and for the second structure even to $M^{2}$. However we lose integrals: On $S T^{*} L^{2}$ we have all two additional integrals, on $S T^{*} M^{2}$ we loose one right-invariant and under projection to $M^{2}$ we loose the other integral. This is consistent with the Kozlov theorem $[\mathrm{Ko}$ according to which the geodesic flow on $M^{2}$ with $g>1$ handles is not analytically integrable. Still we see there is an integrable lift of the flow.

\section{Non-holonomic metrics on suspensions $M_{A}$}

\section{(A) Construction of sub-Riemannian structure.}

Let $M_{A}=T^{2} \tilde{\times}_{A} S^{1}$ be quotient of the cylinder $\mathcal{C}=T^{2}\left(\varphi_{1}, \varphi_{2}\right) \times \mathbb{R}^{1}\left(\varphi_{3}\right)$ w.r.t. the free action given by the map $\hat{A}\left(\varphi_{1}, \varphi_{2}, \varphi_{3}\right)=\left(A\left(\varphi_{1}, \varphi_{2}\right), \varphi_{3}+1\right)$.

$\left.\underline{1}^{\circ}\right)$ Consider at first the case of semisimple $A \in \mathrm{SL}_{2}(\mathbb{Z})$ with real eigenvalues $\lambda>1, \lambda^{-1} \in(0,1)$. Let $\eta_{1}, \eta_{2} \in T_{*}\left(T^{2}\right)$ be the eigenvectors (the case $\lambda=1$ was considered in example 1 and the case $\lambda<0$ will follow similarly because the distribution $\Pi$ has no canonical orientation and so the change $\eta_{j} \mapsto-\eta_{j}$ 
preserves the metrics below). The action on the basis is the following $\hat{A}$ : $\left(\eta_{1}, \eta_{2}, \partial_{\varphi_{3}}\right) \mapsto\left(\lambda \eta_{1}, \lambda^{-1} \eta_{2}, \partial_{\varphi_{3}}\right)$.

We define the contact structure on $\mathcal{C}$ by the vector fields $\xi_{1}=e^{-\ln \lambda \cdot \varphi_{3}} \eta_{1}+$ $e^{\ln \lambda \cdot \varphi_{3}} \eta_{2}, \xi_{2}=\partial_{\varphi_{3}}: \Pi=\left\langle\xi_{1}, \xi_{2}\right\rangle$. Moreover we fix non-holonomic metric $g$ by requiring that $\xi_{1}, \xi_{2}$ is an orthonormal basis.

Lemma 7. $\Pi$ and $g$ are invariant w.r.t the action $\hat{A}$.

Lemma 8. The distribution $\Pi$ is contact.

\section{Proof.}

$$
\left[\xi_{2}, \xi_{1}\right]=\xi_{3}=-\ln \lambda e^{-\ln \lambda \cdot \varphi_{3}} \eta_{1}+\ln \lambda e^{\ln \lambda \cdot \varphi_{3}} \eta_{2} .
$$

But $\xi_{3} \notin \Pi$ because the matrix

$$
\left[\begin{array}{l}
\xi_{1} \\
\xi_{3}
\end{array}\right]=\left(\begin{array}{cc}
e^{-\ln \lambda \cdot \varphi_{3}} & e^{\ln \lambda \cdot \varphi_{3}} \\
-\ln \lambda e^{-\ln \lambda \cdot \varphi_{3}} & \ln \lambda e^{\ln \lambda \cdot \varphi_{3}}
\end{array}\right)
$$

is Vandermonde and hence nondegenerate for $\ln \lambda \neq 0$.

Summarizing we get a sub-Riemannian structure $(\Pi, g)$ on $M_{A}$.

$\left.\underline{\mathbf{2}}^{\circ}\right)$ Now let the eigenvalues of $A$ be $\lambda, \bar{\lambda}=e^{ \pm \theta_{k}}$. Since the matrix is integervalued we have $\theta_{k}= \pm \frac{2 \pi}{k}, k=3,4,6$ (modulo the cases we have considered). Thus $A$ has the matrix $\left(\begin{array}{cc}\cos \theta_{k} & \sin \theta_{k} \\ -\sin \theta_{k} & \cos \theta_{k}\end{array}\right)$ in a basis $\eta_{1}, \eta_{2}$ of $T_{*}\left(T^{2}\right)$. We define $\xi_{1}=\cos \left(\theta_{k} \varphi_{3}\right) \eta_{1}-\sin \left(\theta_{k} \varphi_{3}\right) \eta_{2}, \xi_{2}=\partial_{\varphi_{3}}$ and as before $\Pi=\left\langle\xi_{1}, \xi_{2}\right\rangle$, $g=\left(\xi_{1}^{*}\right)^{2}+\left(\xi_{2}^{*}\right)^{2}$. One easily checks that $(\Pi, g)$ is invariant, non-holonomic and hence defines a sub-Riemannian structure on $M_{A}$.

$\underline{\mathbf{3}}^{\circ}$ ) Consider finally the Jordan box, i.e. $A$ is conjugated to $\left(\begin{array}{ll}1 & 1 \\ 0 & 1\end{array}\right)$. Let $\eta_{1}, \eta_{2}$ be the corresponding basis, $\hat{A}:\left(\eta_{1}, \eta_{2}\right) \mapsto\left(\eta_{1}, \eta_{1}+\eta_{2}\right)$. We set $\xi_{1}=$ $\cos \left(2 \pi \varphi_{3}\right) \eta_{1}+\sin \left(2 \pi \varphi_{3}\right)\left(\eta_{2}-\varphi_{3} \eta_{1}\right), \xi_{2}=\partial_{\varphi_{3}}$ and proceed as before to get sub-Riemannian structure $(\Pi, g)$ on $M_{A}$.

\section{(B) $C^{\infty}$-integrability.}

The Hamiltonian of the sub-Riemannian flow is $H=\frac{1}{2}\left[\xi_{1}^{2}+\xi_{2}^{2}\right]$. Let $p^{\prime}, p^{\prime \prime}, p_{3}$ be momenta dual to vectors $\eta_{1}, \eta_{2}, \partial_{\varphi_{3}}$ from $\mathcal{C}$ (i.e. evaluation matrix $p(\eta)$ is $\left.E_{3 \times 3}\right)$.

This Hamiltonian is $\hat{A}$-invariant, so that it descends to $M_{A}$. Since $H$ does not depend on $\varphi_{1}, \varphi_{2}$ the functions $p_{1}, p_{2}$ and hence $p^{\prime}, p^{\prime \prime}$ are integrals on $\mathcal{C}$. However these functions are not $\hat{A}$-invariant. We get invariants from their combination.

$\left.\underline{1}^{\circ}\right)$ In this case we can write

$$
2 H=\left(e^{\ln \lambda \cdot \varphi_{3}} p^{\prime}+e^{-\ln \lambda \cdot \varphi_{3}} p^{\prime \prime}\right)^{2}+\left(p_{3}\right)^{2} .
$$


Here the functions transform by the rule $\hat{A}:\left(p^{\prime}, p^{\prime \prime}, p_{3}\right) \mapsto\left(\lambda^{-1} p^{\prime}, \lambda p^{\prime \prime}, p_{3}\right)$. Thus we have the following integrals: $I_{1}=H$ and

$$
I_{2}=p^{\prime} p^{\prime \prime}, \quad I_{3}=\sin \left(2 \pi \frac{\ln \left|p^{\prime}\right|}{\ln \lambda}\right) e^{-\left(p^{\prime} p^{\prime \prime}\right)^{-2}},
$$

which are invariant and functionally independent a.e. Thus we conclude $C^{\infty}$ integrability of sub-Riemannian structure $(\Pi, g)$ on $M_{A}$.

$\underline{\mathbf{2}}^{\circ}$ ) In this case the action is the rotation by $\theta_{k}$ and so the additional integrals are:

$$
I_{2}=\left(p^{\prime}\right)^{2}+\left(p^{\prime \prime}\right)^{2}, \quad I_{3}=\operatorname{Re}\left(p^{\prime}+i p^{\prime \prime}\right)^{k} .
$$

Note that in this case the integrals are analytic.

$\left.\underline{\mathbf{3}}^{\circ}\right)$ The action is $\hat{A}:\left(p^{\prime}, p^{\prime \prime}, p_{3}\right) \mapsto\left(p^{\prime}-p^{\prime \prime}, p^{\prime \prime}, p_{3}\right)$. Therefore the additional integrals are:

$$
I_{2}=p^{\prime \prime}, \quad I_{3}=\sin \left(2 \pi \frac{p^{\prime}}{p^{\prime \prime}}\right) e^{-\left(p^{\prime \prime}\right)^{-2}}
$$

This is again the case of $C^{\infty}$, not $C^{\omega}$-integrals.

\section{(C) Obstructions to "nice" integrability.}

Here we generalize Taimanov's result.

Definition. We say sub-Riemannian structure $\left(M^{m}, \Pi, g\right)$ is geometrically simple if $Q=\{H=1 / 2\}$ contains a closed nowhere dense invariant subset $\Gamma$ such that:

1. $Q \backslash \Gamma=\bigcup_{j=1}^{d} U_{j}$, where $U_{j}$ are open and path-connected.

2. Each $U_{j}$ is foliated by Liouville tori, $U_{j} \simeq T^{m} \times D^{m-1}$.

3! Let $\bar{Q}$ be the one-point compactification along fibers of the projection $p$ : $Q \rightarrow M$. Then for every $x \in \bar{Q}$ there exists an arbitrary small neighborhood $W_{x}$ such that $W_{x} \backslash \Gamma$ has a finite number of path-connected components.

The condition $3^{\prime}$ ) is a version of the corresponding 3) from [1] for noncompact $Q$. In fact both conditions are equivalent to the following:

3". For every $x \in M$ there exist arbitrary small neighborhoods $U_{x} \subset V_{x}$ such that $\left|\pi_{0}\left(\left[p^{-1}\left(V_{x} \backslash \bar{U}_{x}\right)\right] \cup\left[p^{-1}\left(\bar{U}_{x}\right) \backslash \Gamma\right]\right)\right|<\infty$.

We remark also that the set $\Gamma$ is usually bigger than the bifurcational set $\Sigma \subset Q$ in Liouville integrable case.

Theorem 9. Let a sub-Riemannian structure $(\Pi, g)$ on a closed manifold $M^{m}$ be Liouville integrable and geometrically simple. Then: 
- The group $\pi_{1}(M)$ is almost commutative, i.e. it contains a commutative subgroup of finite index.

- The first Betti number $\operatorname{dim} H_{1}\left(M^{m} ; \mathbb{R}\right) \leq m$.

Proof. Actually one take a graph with vertices numerated by the components of $p^{-1}\left(V_{x} \backslash \bar{U}_{x}\right) \cup\left(p^{-1}\left(\bar{U}_{x}\right) \backslash \Gamma\right)$. This number is finite due to $\left.3^{\prime \prime}\right)$. Applying sub-Riemannian Hopf-Rinow theorem BR we get labeled directed edges of this graph with labels $s \in \pi_{1}(M, x)$ realized as geodesic loops lifted to $p^{-1}\left(V_{x}\right) \cap(Q \backslash \Gamma)$. The rest of Taimanov's proof [1] goes now unchanged.

We call the set of a.e. independent involutive integrals $I_{1}=H, I_{2}, \ldots, I_{n}$ of Hamiltonian system on $W^{2 n}$ geometrically simple on $Q_{c}=\{H=c\}$ if the bifurcation sets $\Sigma$ are diffeomorphic on $Q_{c^{\prime}}$ for close $c^{\prime} \approx c$ and the stratification of $Q_{c}$ by singularities of the moment map $Q_{c} \ni x \mapsto\left(I_{2}(x) \ldots, I_{n}(x)\right)$ can be extended to stratification $Q_{c}=\cup \tilde{\Sigma}_{j}^{\alpha}$ with $\Gamma=\cup_{j>0} \tilde{\Sigma}_{j}^{\alpha}$ giving the conditions 1-3) of the previous definition. Certainly if sub-Riemannian flow possesses a geometrically simple involutive set of integrals, it is geometrically simple.

For compact $Q$ (Riemannian case) analytic involutive set of integrals is geometrically simple [1]. In sub-Riemannian case non-compactness makes difficulties for application of the standard theorems about sub-analytic (constructive) sets. Yet known integrable cases are geometrically simple. Actually if the integrals are $C^{\omega}$ and polynomial by momenta (in this case we have Liouville tori), the set $\left(I_{j}\right)_{1}^{n}$ is geometrically simple. This is because singularities of $\left(I_{j}\right)$ give stratification of $Q$ at infinity, while the finite (compact) part can be additionally substratified (and even made into simplical decomposition as in [T]).

Corollary 2. Since $\pi_{1}\left(M_{A}\right)$ is not almost commutative for $A$ with real eigenvalues different from \pm 1 and for the Jordan box GK], the sub-Riemannian geodesic flow is not Liouville integrable with geometrically simple set of integrals.

\section{(D) Calculation of topological entropy.}

Calculations of topological entropy in the case of rotation and Jordan box are easy. So we consider only semi-simple case.

First note that the following submanifold $\left\{p^{\prime}=0, p^{\prime \prime}=0, p_{3}=1\right\}$ of $T^{*} \mathcal{C}$ is invariant under the transformation $\hat{A}$. So this 3-dimensional submanifold descends to $T^{*} M_{A}$ and belongs to the hypersurface $\{H=1 / 2\}$. Moreover it is invariant under the geodesic flow and the flow moves with the unit speed along $\partial_{\varphi_{3}}$. So fixing $\varphi_{3}=0$ we get the torus $T^{2}$ and the Poincaré return map of the geodesic flow induces the "Anosov map" $T^{2} \rightarrow T^{2}$ with matrix $A$. Since the entropy of a system is not less than entropy of any subsystem, we conclude: $h_{\text {top }}(\operatorname{sgrad} H) \geq \ln \left|\mathrm{Sp}^{+}(A)\right|$.

Now let us prove the inverse. For this we study bifurcations of $\left(I_{1}, I_{2}, I_{3}\right)$. Let us note that the integrals (7) coincide with the integrals of Bolsinov-Taimanov [BT2], while the Hamiltonians are different. The bifurcation set in the Riemannian case is twice bigger than in the sub-Riemannian one, but still the systems behave similarly. 
It is easy to see that $\operatorname{rk}\left\{d I_{1}, d I_{2}, d I_{3}\right\}=3$ along a trajectory if $\left(p^{\prime}\right)^{2}+\left(p^{\prime \prime}\right)^{2} \neq$ 0 and either $p_{3} \neq 0$ or $e^{\ln \lambda \cdot \varphi_{3}} p^{\prime} \neq e^{-\ln \lambda \cdot \varphi_{3}} p^{\prime \prime}$ at least at one (and then any) point of it. Then by the Liouville theorem this trajectory lies on an invariant torus and the motion is quasi-periodic.

Let $p_{3}=0 \Rightarrow \varphi_{3}=$ const and $p^{\prime \prime}=e^{2 \ln \lambda \cdot \varphi_{3}} p^{\prime}$, but $\left(p^{\prime}\right)^{2}+\left(p^{\prime \prime}\right)^{2} \neq 0$. Then the point moves quasi-periodically along the fiber-torus $T^{2} \subset M_{A}$ and hence again contributes nothing to the topological entropy.

Now on the invariant set $N=\left\{p^{\prime} p^{\prime \prime}=0\right\}$ our Hamiltonian (6) coincides with the one from [BT2]. Therefore the description of the trajectories is the same. In particular $N=N^{\prime} \cup N^{\prime \prime}$, each of the summands $N^{\prime}=\left\{p^{\prime}=0\right\}, N^{\prime \prime}=\left\{p^{\prime \prime}=0\right\}$ being invariant and diffeomorphic to $M_{A} \times S^{1}$. The intersection $N^{\prime} \cap N^{\prime \prime}$ has two components $V^{\prime}, V^{\prime \prime} \simeq M_{A}$ given by $\left\{p_{3}= \pm 1\right\}$ such that $V^{\prime}$ is a stable manifold for $N^{\prime}$ and unstable for $N^{\prime \prime}, V^{\prime \prime}$ is stable for $N^{\prime \prime}$ and unstable for $N^{\prime}$.

Moreover the Hamilton equation of the geodesic flow implies that Lyapunov exponents on $V$ are exactly $\pm \ln \lambda, 0$. So by Katok theorem the only invariant Borel measure on $N$ can be supported on $V^{\prime} \cup V^{\prime \prime}$. But the flow on $V^{\prime}$ and $V^{\prime \prime}$ is Anosov with Poincaré time-one map $A: T^{2} \rightarrow T^{2}$. Therefore the variational principle yields $h_{\text {top }}=\ln \lambda$.

\section{(E) Reeb vector field.}

We prove in this section theorem 2.

The unique (up to sign) contact form $\alpha$ for sub-Riemannian structure is given by the equality $d \alpha\left(\xi_{1}, \xi_{2}\right)=-1$ or $\alpha\left(\left[\xi_{1}, \xi_{2}\right]\right)=1$, where $\xi_{1}, \xi_{2}$ is an arbitrary $g$-orthogonal frame from $C^{\infty}(\Pi)$.

$\left.\underline{1}^{\circ}\right)$ Consider at first the semisimple case $\xi_{1}=e^{-\ln \lambda \cdot \varphi_{3}} \eta_{1}+e^{\ln \lambda \cdot \varphi_{3}} \eta_{2}, \xi_{2}=$ $\partial_{\varphi_{3}}$. The Reeb field satisfies $\nu_{\alpha}=v \bmod \Pi$ for $v=\left[\xi_{1}, \xi_{2}\right]=\ln \lambda\left(e^{-\ln \lambda \cdot \varphi_{3}} \eta_{1}-\right.$ $\left.e^{\ln \lambda \cdot \varphi_{3}} \eta_{2}\right)$.

Lemma 10. $v$ is a symmetry of $\Pi$.

Proof. Actually $\left[v, \xi_{1}\right]=0,\left[v, \xi_{2}\right]=\ln ^{2} \lambda \xi_{1}$.

Corollary 3. $\nu_{\alpha}=v$ is the Reeb vector field.

Proof. Actually $\alpha(v)=1$.

Now we see that the flow of $\nu_{\alpha}$ is quasiperiodic on the tori-fibers of $M_{A}$. Therefore $h_{\mathrm{top}}\left(\nu_{\alpha}\right)$ vanish.

$\underline{\mathbf{2}}^{\circ}$ ) The case of rotation is absolutely similar.

$\left.\underline{\mathbf{3}}^{\circ}\right)$ For the Jordan box the field $v=\left[\xi_{1}, \xi_{2}\right]$ is not a symmetry of $\Pi$ and hence is not the Reeb field. Calculation shows that the contact form is $\alpha=$ $\left(2 \pi+\sin ^{2}\left(2 \pi \varphi_{3}\right)\right)^{-1}\left(\sin \left(2 \pi \varphi_{3}\right) \eta_{1}^{*}+\left(\varphi_{3} \sin \left(2 \pi \varphi_{3}\right)-\cos \left(2 \pi \varphi_{3}\right) \eta_{2}^{*}\right)\right)$, where $\eta_{1}^{*}, \eta_{2}^{*}$ is the co-basis of $T^{*} T^{2}$ dual to $\eta_{1}, \eta_{2}$. Now since $d \alpha=d \varphi_{3} \wedge \partial_{\varphi_{3}}(\alpha)$, the Reeb field is $\nu_{\alpha}=v+b\left(\varphi_{3}\right) \xi_{1}$ and again $h_{\text {top }}\left(\nu_{\alpha}\right)=0$.

Remark 4. Though $h_{\text {top }}(\hat{g})=h_{\text {top }}(g)$ in theorem 月, it is not a simple combination of corollary 1 and theorem 1: formula (3) is not working here. 


\section{Reeb-symmetric SR-structures}

Let $\Pi$ be a contact structure on $M^{2 n+1}$ and $\nu_{\alpha}$ be the Reeb vector field as in introduction.

Definition. Sub-Riemannian structure ( $\Pi, g)$ is called Reeb-symmetric if $\nu_{\alpha}$ is a symmetry of $g$ on $\Pi: L_{\nu_{\alpha}} g=0$. In other words $\nu_{\alpha}$ is a Killing vector field.

For instance the Heisenberg SR-structure (example 2) is of this kind.

Let us define the function $I_{g}(p)=\left\langle p, \nu_{\alpha}\right\rangle^{2}$ (or just $\nu_{\alpha}^{2}$ ) on $T^{*} M$. We will call it Reeb momentum.

Proposition 11. ( $\Pi, g)$ is Reeb symmetric iff the momentum $I_{g}$ is the first integral of the sub-Riemannian geodesic flow.

Proof. Actually locally in Darboux coordinates: $\alpha=d x-\sum_{i=1}^{n} z_{i} d y_{i}$ and $\nu_{\alpha}=\partial_{x}$. Now it's obvious that $L_{\partial_{x}} g=0$ and $\left\{I_{g}, H\right\}=0$ are equivalent to the condition that $g$ does not depend on $x$ [lifted from some $g_{0}$ on $\mathbb{R}^{2 n}(y, z)$ ].

Let us construct by $g$ and $\nu_{\alpha}$ the Riemannian metric $\hat{g}$ on $M$ as in introduction. Then the Riemannian and sub-Riemannian Hamiltonians are connected by the formula

$$
H_{\hat{g}}=H_{g}+I_{g} .
$$

Corollary 4. Reeb momentum $I_{g}$ is an integral of Riemannian geodesic flow if and only if it is an integral for sub-Riemannian one.

Therefore one can integrate instead of $g$, the metric $\hat{g}$, finding involutive set of integrals starting from $I_{1}=H_{\hat{g}}$ and $I_{2}=I_{g}$.

Remark 5. Let us note that the metrics $\hat{g}^{(t)}$, which equal $g$ on $\Pi$ and $1 / \sqrt{t}$ on orthogonal $\nu_{\alpha}$, satisfy: $\lim _{t \rightarrow+0} \operatorname{dist}_{\hat{g}^{(t)}}=\operatorname{dist}_{g}-$ sub-Riemannian distance. The corresponding Hamiltonians $H_{\hat{g}^{(t)}}=H_{g}+t I_{g}$.

Let us note also about topological entropy:

Proposition 12. If $(\Pi, g)$ is smooth and Reeb-symmetric, then vanishing of two of three $h_{\text {top }}\left(\operatorname{sgrad} H_{\hat{g}}\right), h_{\text {top }}\left(\operatorname{sgrad} H_{g}\right), h_{\text {top }}\left(\nu_{\alpha}\right)$ implies vanishing of the third. Moreover if $h_{\text {top }}\left(\nu_{\alpha}\right)=0$, then $h_{\text {top }}(g)=h_{\text {top }}(\hat{g})$.

Proof. Actually this follows from (9) and commutativity.

Remark 6. Note that even though the Reeb field $\nu_{\alpha}$ is divergence-free and shares some good properties (no attractors/repellers), we can have $h_{\text {top }}\left(\nu_{\alpha}\right)>0$. Examples are provided by geodesic flows on negatively curved manifolds. 


\section{Concluding remark: norm via entropy}

Any integrable Hamiltonian system on $\left(M^{2 n}, \omega\right)$ with integrals $\left(I_{1}, \ldots, I_{n}\right)$ determines $\mathbb{R}^{n}$-action with generators $\varphi_{j}^{t_{j}}$ being shifts along $\operatorname{sgrad}_{\omega}\left(I_{j}\right)$. Then the formula (5) with $h=h_{\text {top }}$ gives a pseudonorm $\rho=\rho_{\text {top }}$ on this $\mathbb{R}^{n}$ (of course for $h=h_{\mu}$ with Liouville invariant measure $\mu$ we have $\rho_{\mu} \equiv 0$ ).

For instance if the action reduces to the action of the torus $T^{n}$ then $\rho_{\text {top }} \equiv 0$. This is obvious from the definition (also is seen from Atiyah convexity theorem).

Examples of this and [BT2] papers provide $\mathbb{R}^{3}$-action with $\rho_{\text {top }} \not \equiv 0$. However $\rho_{\text {top }}$ is not a norm, because $h_{\text {top }}(\operatorname{sgrad} I)=0$ for any $I=\lambda_{2} I_{2}+\lambda_{3} I_{3}$.

Theorem 13. There is an integrable Hamiltonian system with nondegenerate $\rho_{\text {top }}$ (norm).

To explain the result consider a piece of bifurcation diagram for the momentum map $\left(I_{1}, I_{2}, I_{3}\right)$ in the semisimple case that is pictured in Fig. 1. The central vertical line corresponds to the most complicated singularity $\left\{p_{3}= \pm 1\right\}$ and in the preimage of each of its point we have 2 copies of $M_{A}$. Two curved boundary surfaces correspond to bifurcation diagram either, while 4 other plane pieces of the boundary consist of regular points and appear due to the size of a neighborhood.

Fig. 1

Now let's take 3 mutually non-intersecting such pieces in the affine $\mathbb{R}^{3}$ such that their central axes are pair-wise non-parallel (we can direct them along axes $\left.I_{1}, I_{2}, I_{3}\right)$. We connect their regular points by small tubes as shown in Fig. 2. 
Fig. 2

This is equivalent to connecting preimages of pieces by the tubes $T^{3} \times I \times D^{2}(\varepsilon)$. The tubes in $\mathbb{R}^{3}$ carry the affine structure, that permits by Arnold-Liouville theorem to define symplectic form and a Hamiltonian on the obtained manifold which is integrable with the Hamiltonian action of $\mathbb{R}^{3}$ given by $\left(I_{1}, I_{2}, I_{3}\right)$ and such that $\rho_{\text {top }}$ is a norm on this $\mathbb{R}^{3}$. 


\section{References}

[A] V.I. Arnold, "Mathematical methods of classical mechanics", Nauka, Moscow (1989); Engl. transl. in Graduale Texts in Mathematics, Springer

[ACG] A. A. Agrachev, El-A., El-H. Chakir, J. P. Gauthier, "Sub-Riemannian metrics on $\mathbb{R}^{3}$, Geometric control and non-holonomic mechanics (Mexico City, 1996), CMS Conf. Proc., 25, A.M.S., Providence, RI, (1998), 29-78

[AS] A. A. Agrachev, A. V. Sarychev, "Sub-Riemannian metrics: minimality of abnormal geodesics versus subanalyticity", ESAIM Control Optim. Calc. Var. 4 (1999), 377-403

[AKN] V.I. Arnold, V.V. Kozlov, A.I. Neishtadt, "Mathematical aspects of classical and celestial mechanics", in Dynamical systems III, Encyclopaedia of Mathematical Sciences 3; Springer-Verlag, Berlin (1997)

[B] L. Butler, "A new class of homogeneous manifolds with Liouvilleintegrable geodesic flows", prep. 1998-8, Queen's Univ. at Kingston, Canada (1998)

[BR] "Sub-Riemannian geometry", A. Belaïche, J.-J. Risler Ed., Birkhäuser-Verlag (1996)

[BF] A. V. Bolsinov, A. T. Fomenko, "Integrable Geodesic Flows on TwoDimensional Surfaces", (Monographs in Contemporary Mathematics) Consultants Bureau, New York (2000)

[BT1] A. V. Bolsinov, I. A. Taimanov, "Integrable geodesic flows on the suspensions of toric automorphisms", e-print: http://xxx.lanl.gov/abs/math.DG/9911193 (1999)

[BT2] A. V. Bolsinov, I. A. Taimanov, "Integrable geodesic flows with positive topological entropy", Invent. Math. 140, no. 3 (2000), 639-650

[F] A. T. Fomenko, "Symplectic geometry", Gordon and Breach Publ. (1995)

[GK] R.I. Grigorchuk, P.F. Kurchanov, "Some problems in group theory that are connected with geometry", in Algebra VII, Encyclopaedia of Mathematical Sciences 58; Springer-Verlag, Berlin (1993)

[Hsu] L. Hsu, "Calculus of variations via the Griffiths formalism", J. Differential Geom. 36, no. 3 (1992), 551-589

$[\mathrm{Hu}] \mathrm{HuYi} \mathrm{Hu}$, Some ergodic properties of commuting diffeomorphisms", Ergodic Theory Dynam. Systems 13, no. 1 (1993), 73-100 
[KH] A. Katok, B. Hasselblatt, "Introduction to the modern theory of dynamical systems", Encyclopedia of Math. and its Appl. 54, Cambridge University Press, Cambridge (1995)

[Ko] V.V. Kozlov, "Topological obstacles to the integrability of natural mechanical systems", Soviet Math. Dokl. 20 (1979), 1413-1415

$[\mathrm{Kr}] \quad$ B.S. Kruglikov, "Symplectic and contact Lie algebras with an application to Monge-Ampère equations" Proc. Steklov Inst. Math. 221 (1998), 221-235

[MSS] R. Montgomery, M. Shapiro, A. A. Stolin, "A nonintegrable subRiemannian geodesic flow on a Carnot group", J. Dynam. Control Systems 3, no. 4 (1997), 519-530

[Pa] K. K. Park, "On directional entropy functions", Israel Journal of Math. 113 (1999), 243-267

[PBGM] L. S. Pontrjagin, V.G. Boltyanskii, R. V. Gamkrelidze, E. F. Mishchenko, "The mathematical theory of optimal processes", Wiley, Interscience (1962)

[R] P.K. Rashevsky, "Any two points of a totally nonholonomic space may be connected by an admissible line", Usp. Zap. Ped. Inst. im. Liebknechta, Ser. Phys. Math. 2 (1938), 83-94 (Russian)

[S] Ja.G. Sinai, "Topics in Ergodic theory", Princeton Univ. PRess, Princeton NJ (1994)

[T] I. A. Taimanov, "Topology of Riemannian manifolds with integrable geodesic flows", Proc. Steklov Inst. Math. 205 (1995), 139-150

[VG] A.M. Vershik, V. Ya. Gershkovich, "Nonholonomic dynamical systems. Geometry of distributions and variational problems", in Dynamical Systems VII, V. I. Arnold, S. P. Novikov (Eds.), Encyclopaedia of Math. Sciences 16, Springer (1994)

Inst. of Math., University of Tromsoe, Tromsoe 90-37, Norway; kruglikov@math.uit.no 
This figure "bifdgr01.gif" is available in "gif" format from: http://arXiv.org/ps/math/0105128v2 
This figure "bifdgr02.gif" is available in "gif" format from: http://arXiv.org/ps/math/0105128v2 